\title{
Platelet-rich plasma in orthopaedics - to use or not to use?
}

\author{
Antun Šumanovac \\ Department of Anatomy and Neuroscience, JJ Strossmayer University of Osijek, Croatia \\ Department of Orthopaedics and Traumatology, County General Hospital Vinkovci, Croatia
}

Correspondence: Antun Šumanovac, Department of Anatomy and Neuroscience, Faculty of Medicine, JJ Strossmayer University of Osijek, Cara Hadrijana IOE HR-3 I000 Osijek Croatia, ORCID: 0000-000I-9I73-6686,

Department of Orthopaedics and Traumatology, County General Hospital Vinkovci, Zvonarska 57 HR-32 I00 Vinkovci, Croatia, Email antun. sumanovac@gmail.com

Received: March 22, 2018 | Published: March 23, 2018

Copyright@ 2018 Šumanovac. This is an open access article distributed under the terms of the Creative Commons Attribution License, which permits unrestricted use, distribution, and reproduction in any medium, provided the original author and source are credited.

\begin{abstract}
There is a clear shift in modern medicine towards non-operative treatment or minimally invasive treatment of diseases. Orthopaedic surgery is no exception. Patients demand quick return to activity levels prior to injury and biological treatment is becoming more and more mainstream. PRP is one of the most studied orthobiologic agents in orthopaedics and is becoming more widely used. But the question is, should we encourage widespread usage of PRP at this time?
\end{abstract}

\section{Introduction}

Platelet-rich plasma (PRP) is one of the most studied orthobiologic agents. Orthobiologics are substances found in the human body that can be used to improve healing of cartilage, muscles, ligaments, tendons and fractures. ${ }^{1}$ These agents can be directly injected in the injured structure or used as intraarticular injection thus avoiding systemic effects of drugs in conservative treatment and increasing the bioavailability of the desired treatment. In recent years, pressure on orthopaedic surgeons has risen to enable quick return to pre-injury level of activity. The former is today not only true for professional athletes but increasing elderly population also wishes to remain active. More effective and minimally invasive treatment options are actively being sought. Usage of PRP, especially in private practice, is on the rise. Should we encourage it's use or cautiously back further research?

\section{PRP}

We've all been there - a patient comes to our office either with a distinct sports related injury of the locomotor system or a degenerative disease. Wouldn't it be great if we could simply take a sample of his blood, have it processed in a laboratory and as such inject it at the site of injury, resulting in a fast, cost-effective healing of the injury? That's where PRP is supposed to come in. The appeal of PRP is it's high concentration of growth factors that are involved in the healing process. Furthermore, the components found in PRP preparations, cellular and chemical, are numerous. PRP is actually a milieu of factors that are both catabolic/anabolic and also pro-/antiinflammatory. ${ }^{2}$ That complexity makes it difficult to reproduce precise elements in each patient and company's product. ${ }^{2}$ A key point here is the cellular composition of PRP - increasing platelet concentrations are associated with anabolic signaling while increased leukocyte concentrations promote catabolic signaling. ${ }^{3}$ Keeping all that in mind, it is no wonder that there is no standardized method or device for PRP production. Furthermore, injection frequency and volume vary widely among published studies. Variables include platelet concentration, leukocyte concentration, use of anticoagulants, use of platelet pre-activation factors and injection volume and frequency. ${ }^{4}$ In order to produce PRP, blood must be drawn during each visit since it can not be stored or frozen which can also contribute to variability in composition between blood withdrawals and results. ${ }^{5}$

\section{The road so far}

Published reviews and meta-analyses draw variable conclusions in regard to clinical efficacy of PRP. Most of the literature reported PRP to be beneficial for a short period of time in knee osteoarthritis $(\mathrm{OA}){ }^{6}$ Campbell et al. found that PRP injections in early-stage OA was an effective treatment for up to 12 months. $^{7}$ Another systematic review found that current studies are inconclusive regarding efficacy of PRP. ${ }^{8}$ Some studies concluded that PRP is associated with improved efficacy compared with hyaluronic acid (HA) and placebo. ${ }^{9}$ It is necessary to note that concerns exist regarding the quality of published papers and the high risk of bias. ${ }^{10}$ The PRP treatment is not without problems. A meta-analysis of PRP papers found an increased incidence of nonspecific adverse effects among patients treated with PRP when compared to HA and placebo. ${ }^{9}$ One review noted increased local adverse reactions associated with serial PRP injections. ${ }^{7}$ There is also a question of leukocyte concentration in PRP preparations since increasing the concentration of leukocytes in intraarticular space can promote inflammation. A meta-analysis on randomized control trials 
comparing clinical outcomes and rates of adverse events between leukocyte-poor (LP) and leukocyte-rich (LR) PRP found 3 trials that used LP PRP which reported positive effects compared with HA. Only one trial using LR PRP reported positive effects compared to HA. ${ }^{11}$ Polymorphonuclear neutrophils may have a negative effect on cartilage, increasing existing tissue damage.

\section{So, what's next?}

It is clear that PRP has possible benefits in treatment of musculoskeletal disorders. However, it is also clear that there is no consensus on optimal protocol of preparing PRP and products used have different compositions. That makes it extremely difficult to compare different studies and for orthopaedic societies to give recommendations regarding PRP. It is clear that there is a need to standardize preparation protocols and to make composition differences as low as possible.

\section{Conclusion}

Although advencements have been made in the field of orthobiologics, these agents are still in their beginnings. In order to make further improvements, it is necessary to define minimal standards for such treatments. Studies on PRP are a clear example of that. High number of variables require detailed preparation protocols. Future randomized trials should focus on larger sample sizes and longer follow-up times for the evidence to be more compelling. Without such studies, orthopaedic societies cannot give their recommendations on usage of PRP. Furthermore, better defined controls are also needed in order to decrease bias of the studies to a minimum level.

It is my opinion that PRP still does not warrant usage outside of controlled research environment until we have well defined protocols and reproducible studies. PRP usage is not without possible adverse effects and long-term effects of it's usage are still unclear.

\section{References}

1. Sampson SE, Vincent H, Ambach MA. Education and Understanding Orthobiologics: Then and Now. In: Gobbi A, Espregueira-Mendes J, Lane JG, et al. Bio-orthopaedics. Berlin, Heidelberg: Springer Verlag. 2017;p.41-46.
2. Boswell SG, Cole BJ, Sundman EA, et al. Platelet-rich plasma: a milieu of bioactive factors. Arthroscopy. 2012;28(3):429-439.

3. Sundman EA, Cole BJ, Fortier LA. Growth factor and catabolic cytokine concentrations are influenced by the cellular composition of platelet-rich plasma. Am J Sports Med. 2011;39(10):2135-2140.

4. Wehling P, Evans C, Wehling J, et al. Effectiveness of intra-articular therapies in osteoarthritis: a literature review. Ther Adv Musculoskelet Dis. 2017;9(8):183-196.

5. Mazzocca AD, McCarthy MBR, Chowaniec DM, et al. Platelet-Rich Plasma Differs According to Preparation Method and Human Variability. J Bone Joint Surg Am. 2012;94(4):308-316.

6. Dhillon M, Patel S, Bali K. Platelet-rich plasma intra-articular knee injections for the treatment of degenerative cartilage lesions and osteoarthritis. Knee Surgery Sport Traumatol Arthrosc. 2011;19(5):863864.

7. Campbell KA, Saltzman BM, Mascarenhas R, et al. Does Intra-articular Platelet-Rich Plasma Injection Provide Clinically Superior Outcomes Compared With Other Therapies in the Treatment of Knee Osteoarthritis? A Systematic Review of Overlapping Meta-analyses. Arthroscopy. 2015;31(11):2213-2221.

8. Lai LP, Stitik TP, Foye PM, et al. Use of Platelet-Rich Plasma in IntraArticular Knee Injections for Osteoarthritis: A Systematic Review. PM R. 2015;7(6):637-648.

9. Khoshbin A, Leroux T, Wasserstein D, et al. The Efficacy of PlateletRich Plasma in the Treatment of Symptomatic Knee Osteoarthritis: A Systematic Review with Quantitative Synthesis. Arthroscopy. 2013;29(12):2037-2048.

10. Dold AP, Zywiel MG, Taylor DW, et al. Platelet-Rich Plasma in the Management of Articular Cartilage Pathology. Clin J Sport Med. 2014;24(1):31-43.

11. Riboh JC, Saltzman BM, Yanke AB, et al. Effect of Leukocyte Concentration on the Efficacy of Platelet-Rich Plasma in the Treatment of Knee Osteoarthritis. Am J Sports Med. 2016;44(3):792-800. 